\title{
Ketoprofen in the Cat: Pharmacodynamics and Chiral Pharmacokinetics
}

\begin{abstract}
The non-steroidal anti-inflammatory drug ketoprofen (KTP) was administered as the racemate to cats intravenously (IV) and orally at clinically recommended dose rates of 2 and $1 \mathrm{mg} / \mathrm{kg}$, respectively, to establish its chiral pharmacokinetic and pharmacodynamic properties. After IV dosing, clearance was more than five times greater and elimination halflife and mean residence time were approximately three times shorter for R(-) KTP than for $\mathrm{S}(+)$ KTP. Absorption of both $\mathrm{S}(+)$ and $\mathrm{R}(-)$ enantiomers was rapid after oral dosing and enantioselective pharmacokinetics was demonstrated by the predominance of $\mathrm{S}(+) \mathrm{KTP}$, as indicated by plasma AUC of $20.25(\mathrm{~S}(+) \mathrm{KTP})$ and $4.09(\mathrm{R}(-) \mathrm{KTP}) \mu \mathrm{g} \mathrm{h} / \mathrm{mL}$ after IV and $6.36(\mathrm{~S}(+) \mathrm{KTP})$ and $1.83(\mathrm{R}(-) \mathrm{KTP}) \mu \mathrm{g} \mathrm{h} / \mathrm{mL}$ after oral dosing. Bioavailability after oral dosing was virtually complete. Reduction in ex vivo serum thromboxane (TX)B2 concentrations indicated marked inhibition of platelet cyclo-oxygenase (COX)-1 for $24 \mathrm{~h}$ after both oral and IV dosing and inhibition was statistically significant for $72 \mathrm{~h}$ after IV dosing. Both oral and IV rac-KTP failed to affect wheal volume produced by intradermal injection of the mild irritant carrageenan but wheal skin temperature was significantly inhibited by IV rac-KTP at some recording times. Possible reasons for the disparity between marked COX-1 inhibition and the limited effect on the cardinal signs of inflammation are considered. In a second experiment, the separate enantiomers of KTP were administered IV, each at the dose rate of $1 \mathrm{mg} / \mathrm{kg}$. S(+)KTP again predominated in plasma and there was unidirectional chiral inversion of $\mathrm{R}(-)$ to $\mathrm{S}(+) \mathrm{KTP}$. Administration of both enantiomers again produced marked and prolonged inhibition of platelet COX-1 and, in the case of R(-)KTP, this was probably attributable to $\mathrm{S}(+) \mathrm{KTP}$ formed by chiral inversion.
\end{abstract}

Keyword: Ketoprofen, cat, pharmacodynamics, pharmacokinetics, enantiomers, chirality 